\title{
Anestesia intravenosa contínua com cetamina racêmica ou dextrocetamina e detomidina em cadelas
}

[Continuous intravenous anesthesia with racemic ketamine or dextroketamine and detomidine in female dogs]

\author{
F.V. Henrique ${ }^{1}$, S.A.R.S. Pereira ${ }^{2}$, L.F. Batista ${ }^{2}$, L.H. Oliveira $^{2}$, N.M.O. Monteiro ${ }^{1}$, \\ R.N. Parentoni ${ }^{3}$, A.P. Souza ${ }^{4}$, A.F.M. Vaz ${ }^{4}$, P.I. Nóbrega Neto ${ }^{4}$ \\ ${ }^{1}$ Médica veterinária autônoma \\ ${ }^{2}$ Aluno de pós-graduação - Universidade Federal de Campina Grande - Patos, PB \\ ${ }^{3}$ Técnica administrativa - Universidade Federal da Paraíba - João Pessoa, PB \\ ${ }^{4}$ Universidade Federal de Campina Grande - Patos, PB
}

\begin{abstract}
RESUMO
Objetivou-se com este estudo comparar a associação de detomidina e cetamina ou dextrocetamina, por via intravenosa contínua, em oito cadelas submetidas a dois protocolos: GCD - indução anestésica com $5 \mathrm{mg} / \mathrm{kg}$ e infusão intravenosa contínua de $20 \mathrm{mg} / \mathrm{kg} / \mathrm{h}$ de cetamina; e GDD - indução com 3,5mg/kg e infusão de $14 \mathrm{mg} / \mathrm{kg} / \mathrm{h}$ de dextrocetamina. Associou-se detomidina, $30 \mu \mathrm{g} / \mathrm{kg} / \mathrm{h}$, em ambos os grupos. Registraram-se frequência cardíaca (FC), pressão arterial (PA), frequência respiratória ( $f$ ), temperatura (TC), miorrelaxamento, analgesia, hemogasometria e eletrocardiograma, antes e 15 minutos após a MPA (Mbasal e Mmpa); após o início da infusão (Mic); a cada 10 minutos até 90 minutos (M10, M20, M30, M40, M50, M60, M70, M80 e M90); e 30 minutos após o fim da infusão (M120). Foi observada bradicardia em Mmpa no GCD e de Mmpa a M10 no GDD. Ocorreu hipotensão em Mmpa e hipertensão a partir de Mic. A $f$ diminuiu de M10 a M30. Foram observados: onda T de alta amplitude, bloqueios atrioventriculares e parada sinusal. Ocorreu acidose respiratória. O período de recuperação foi de $219,6 \pm 72,3$ minutos no GCD e de 234,1 $\pm 96,8$ minutos no GDD. A cetamina e a dextrocetamina, associadas à detomidina por infusão contínua, causam efeitos cardiorrespiratórios e anestésicos similares.
\end{abstract}

Palavras-chave: agonista $\alpha 2$-adrenérgico, antagonista NMDA, canino, cetamina $\mathrm{S}(+)$

\begin{abstract}
The combination of detomidine and ketamine or dextrocetamine for continuous intravenous infusion was compared in eight female dogs submitted to two protocols: GCD - 5mg/kg of anesthetic induction and continuous intravenous infusion of ketamine $20 \mathrm{mg} / \mathrm{kg} / \mathrm{h}$; and GDD - induction with $3.5 \mathrm{mg} / \mathrm{kg}$ and infusion of $14 \mathrm{mg} / \mathrm{kg} / \mathrm{h}$ of dextrocetamine. Detomidine, $30 \mu \mathrm{g} / \mathrm{kg} / \mathrm{h}$ was associated in both groups. Heart rate (HR), blood pressure $(B P)$, respiratory rate $(R R)$, temperature $(C T)$, myorelaxation, analgesia, blood gas analysis and electrocardiogram were recorded before and 15 minutes after MPA (Mbasal and Mmpa); after the start of infusion (Mic); every 10 minutes to 90 minutes (M10, M20, M30, M40, M50, M60, M70, M80 and M90); and 30 minutes after the end of infusion (M120). Bradycardia was observed in Mmpa in GCD and from Mmpa to M10 in GDD. There was hypotension in Mmpa and hypertension from Mic. The RR decreased from M10 to M30. High amplitude T wave, atrioventricular blocks and sinus arrest were observed. Respiratory acidosis occurred. The recovery period was $219.6 \pm 72.3$ minutes in GCD and 234.1 \pm 96.8 minutes in GDD. Ketamine and $S+$ ketamine associated with detomidine for continuous infusion cause cardiorespiratory and similar anesthetic effects.
\end{abstract}

Keywords: $\alpha 2$-adrenergic agonist, NMDA antagonist, canine, ketamine $S(+)$

Recebido em 10 de outubro de 2019

Aceito em 17 de outubro de 2020

E-mail: nandinhavh@gmail.com 


\section{INTRODUÇÃO}

A cetamina pode ser usada clinicamente na forma racêmica ou como isômero levorrotatório (cetamina $\mathrm{S}+$ ), denominado dextrocetamina. Esse isômero tem sido utilizado em cães como uma alternativa mais potente à cetamina racêmica, sendo a cetamina $S(+)$ 1,5 vez mais potente nessa espécie (Casoni et al., 2015). A detomidina, um agonista $\alpha 2$-adrenérgico, é um potente miorrelaxante, com ação sedativa e analgésica, comumente utilizada em equinos (Pohl et al., 2012), não sendo muito difundida em pequenos animais.

Henrique et al. (2019), ao avaliarem a associação de detomidina e dextrocetamina por via intravenosa contínua, em cadelas submetidas à ovário-histerectomia $(\mathrm{OH})$, observaram depressão cardiovascular e respiratória. Objetivou-se avaliar os efeitos cardiorrespiratórios e anestésicos, bem como a qualidade da anestesia promovida pela associação de detomidina e cetamina racêmica ou dextrocetamina por via intravenosa contínua em cadelas, e apresentar dados que possam contribuir na aplicabilidade de tais protocolos em procedimentos cirúrgicos na espécie canina.

\section{MATERIAL E MÉTODOS}

Após a aprovação da Comissão de Ética (protocolo 286/2015), foram utilizadas oito cadelas sem raça definida, hígidas, com $3 \pm 1$ anos e $14,8 \pm 2,4 \mathrm{~kg}$. Os animais foram alojados em canis individuais, em área arejada, onde permaneceram por sete dias recebendo alimentação à base de ração comercial e água ad libitum. Anteriormente ao experimento, realizou-se jejum alimentar de 12 horas e hídrico de quatro horas e tricotomia da região do terço médio dorsal do antebraço e da região dorsal da orelha. Todos os animais foram submetidos a dois protocolos, com intervalo de 15 dias. $\mathrm{O}$ experimento era realizado em sala climatizada com temperatura de $23^{\circ} \mathrm{C}$. Foram compostos aleatoriamente dois grupos, nos quais os animais foram tratados com os seguintes fármacos:

grupo cetamina racêmica + detomidina (GCD): administrou-se, por via intramuscular (IM), $0,02 \mathrm{mg} / \mathrm{kg}$ de detomidina (Dormiun $\mathrm{V}^{\circledR}$, Agener União, Brasil) e, após 15 minutos, um bolus intravenoso de $5 \mathrm{mg} / \mathrm{kg}$ de cetamina racêmica (Cetamin ${ }^{\circledR}$, Syntec, Brasil), seguido de infusão intravenosa contínua do mesmo fármaco, na dose de $20 \mathrm{mg} / \mathrm{kg} / \mathrm{h}$, associado à detomidina, na dose de $30 \mu \mathrm{g} / \mathrm{kg} / \mathrm{h}$; grupo dextrocetamina + detomidina (GDD): administrou-se, por via IM, 0,02 mg/ $\mathrm{kg}$ de detomidina e, após 15 minutos, um bolus intravenoso de $3,5 \mathrm{mg} / \mathrm{kg}$ de dextrocetamina (Ketamin ${ }^{\circledR}$, Cristália Produtos Farmacêuticos Ltda., Brasil), seguido de infusão intravenosa contínua do mesmo fármaco, na dose de $14 \mathrm{mg} / \mathrm{kg} / \mathrm{h}$, associado à detomidina, na dose de $30 \mu \mathrm{g} / \mathrm{kg} / \mathrm{h}$.

Implantou-se um cateter $22 \mathrm{G}$ na veia cefálica, por onde os fármacos foram infundidos, empregandose uma torneira de três vias e uma bomba de infusão peristáltica (Bomba de infusão peristáltica rotativa MP-20 ${ }^{\circledR}$ - CELM, Stramedical, Brasil) para cada fármaco. Introduziu-se um cateter $24 \mathrm{G}$ na artéria auricular média esquerda para a coleta de amostras de sangue para hemogasometria e para a mensuração da pressão arterial. Após a MPA, o animal foi colocado em decúbito lateral direito sobre uma mesa cirúrgica revestida com colchão, onde foi realizado o bolus do fármaco correspondente ao grupo, seguido do início da infusão intravenosa contínua dele, associado à detomidina, por um período de 90 minutos. Foram considerados os seguintes momentos: imediatamente antes e 15 minutos após a MPA (Mbasal e Mmpa); imediatamente após o início da infusão contínua (Mic); a cada 10 minutos a partir desta, por um período de 90 minutos (M10, M20, M30, M40, M50, M60, M70, M80 e M90); e 30 minutos após o fim da infusão (M120).

Foram avaliados: frequência cardíaca (FC), em batimentos por minuto, por meio de um eletrocardiógrafo computadorizado (DL 1000 ${ }^{\circledR}$, Deltalife, Brasil), calculando-se a duração entre dois intervalos R-R, em milissegundos; pressão arterial média (PAM), em $\mathrm{mmHg}$, a partir da conexão de um esfigmomanômetro aneroide à artéria auricular média previamente cateterizada, mantendo-se a interface líquido-ar do sistema à altura do manúbrio esternal; frequência respiratória $(f)$ pela observação dos movimentos respiratórios, durante um minuto $(\mathrm{mpm})$; e temperatura corporal (TC) mensurada com termômetro clínico digital (TH186-G ${ }^{\circledR}-\mathrm{Tech}$, Brasil) inserido no ânus do animal.

Efetuou-se o eletrocardiograma (ECG) em derivação DII com emprego de eletrocardiógrafo computadorizado, pelo qual foram observadas, 
em milissegundos (ms) ou milivolts $(\mathrm{mV})$, a duração (Pms) e a amplitude da onda P (PmV), a duração do complexo QRS (QRSms), a amplitude da onda $\mathrm{R}(\mathrm{RmV})$ e a duração dos intervalos entre as ondas Q e T (QTms) e P e R (PRms). Também foi registrada a presença de arritmias cardíacas. As amostras de sangue arterial $(0,5 \mathrm{~mL})$ para hemogasometria foram coletadas através do cateter colocado na artéria auricular, em seringas de insulina heparinizadas. O sangue coletado foi processado imediatamente em analisador automático de gases sanguíneos (Analisador de pH e gases sanguíneos AGS 22 digital $^{\circledR}$, Brasil).

Foram mensurados o $\mathrm{pH}$, a pressão parcial de oxigênio $\left(\mathrm{PaO}_{2}\right.$, em $\left.\mathrm{mmHg}\right)$, a pressão parcial de dióxido de carbono $\left(\mathrm{PaCO}_{2}\right.$, em $\left.\mathrm{mmHg}\right)$, a saturação de oxi-hemoglobina $\left(\mathrm{SaO}_{2}, \quad\right.$ em percentual), a concentração de bicarbonato $\left(\mathrm{HCO}_{3}{ }^{-}\right.$, em mmol/L), o excesso/déficit de base (EB, em $\mathrm{mmol} / \mathrm{L}$ ) e o dióxido de carbono total $\left(\mathrm{TCO}_{2}\right.$, em $\left.\mathrm{mmol} / \mathrm{L}\right)$. A hemogasometria foi realizada em Mbasal, Mmpa, M30, M60, M90 e M120. Para a avaliação do grau de analgesia, foi utilizada metodologia adaptada de Figueiró et al. (2016), empregando-se um estimulador elétrico (Eletroestimulador MT-10 ${ }^{\circledR}$, Medcir, Brasil) conectado a um par de agulhas de aço inoxidável distanciadas entre si por $3 \mathrm{~cm}$ e inseridas subcutaneamente na base ventral da cauda.

Cada estímulo $(30 \mathrm{~Hz}, 400 \mu \mathrm{s})$ foi aplicado aumentando-se a corrente constante (em mA) até que uma resposta positiva foi observada (movimentos de cabeça ou membros). Três observadores avaliaram a resposta. Em caso de desacordo entre eles, a resposta foi descartada, e o estímulo repetido. Essa avaliação foi realizada a partir do Mic até o M120. Avaliou-se o miorrelaxamento pelo grau de rigidez e resistência dos membros à manipulação e pelo tono muscular: excelente (escore 2) - total flacidez muscular; bom (escore 1) - moderada manutenção do tono muscular com tremores; e ruim (escore 0) - tremores e rigidez, catalepsia ou movimentação intensa. $\mathrm{O}$ miorrelaxamento foi avaliado nos mesmos momentos da avaliação paramétrica.

A duração da recuperação foi considerada como o tempo, em minutos, decorrido entre o final da infusão até o retorno da deambulação. A qualidade da recuperação foi classificada como: excelente (escore 2) - repouso tranquilo; boa (escore 1) - moderada excitação; ou ruim (escore
0) - agitação, tremores, mioclonias e/ou convulsões. As classificações para miorrelaxamento e recuperação foram adaptadas de Cardoso et al. (2008). A análise estatística foi realizada empregando-se o programa BioEstat 5.0 ao nível de 5\% de significância. Primeiramente, os dados foram submetidos ao teste de normalidade de Shapiro-Wilk. Utilizou-se a análise de variância de duas vias e o teste de Tukey ou o teste de Friedman. A comparação entre os grupos foi realizada pelo teste $t$ de Student ou U-Mann-Withney. Os dados de miorrelaxamento foram analisados pelo teste de Friedman e U-Mann-Whitney e são apresentados como mediana (valor máximo - valor mínimo). Os dados paramétricos são apresentados como média \pm desvio-padrão, e os não paramétricos como mediana \pm desvio interquartílico.

\section{RESULTADOS E DISCUSSÃO}

As doses utilizadas neste trabalho foram baseadas no estudo de Silva et al. (2007), que compararam a infusão contínua de midazolam $(0,4 \mathrm{mg} / \mathrm{kg} / \mathrm{h})$, cetamina $(20 \mathrm{mg} / \mathrm{kg} / \mathrm{h})$ e xilazina $(1 \mathrm{mg} / \mathrm{kg} / \mathrm{h})$ ao mesmo protocolo, porém substituindo a xilazina pela medetomidina $(30 \mu \mathrm{g} / \mathrm{kg} / \mathrm{h})$, em cadelas submetidas à cirurgia de ovário-histerectomia. Além disso, segundo Casoni et al. (2015) a cetamina $\mathrm{S}(+)$ é $1,5 \mathrm{vez}$ mais potente na espécie canina, daí a dose de dextrocetamina utilizada neste experimento.

Após a MPA e antes de induzir a anestesia com a cetamina racêmica ou dextrocetamina, três animais, os mesmos em ambos os grupos, apresentavam-se em decúbito lateral, mas, ao serem colocados na mesa, mostravam-se acordados e interagiam com os pesquisadores. Cinco animais permaneceram em decúbito esternal até serem posicionados em decúbito lateral direito. Não houve excitação e alterações comportamentais em nenhuma cadela após a MPA. Por se tratar de uma anestesia dissociativa, nenhum animal apresentou perda de reflexos oculares e o globo ocular permaneceu centralizado durante toda a infusão anestésica, em ambos os grupos. Porém, nenhum animal tentou se levantar ou manifestou sinais de excitação durante a anestesia. Como o intuito do experimento era avaliar a anestesia promovida por ambos os protocolos estudados, em nenhum momento se considerou o aumento da dose dos fármacos administrados. 
Houve uma redução na FC no GCD, em relação ao Mbasal em Mmpa, M10, M20, M30, M40 e M60, havendo ainda diferença significativa entre Mmpa e Mbasal, Mic, M80 e M90 (Tab. 1). A redução na FC após a MPA pode ser atribuída aos efeitos cardiovasculares depressores da detomidina (Ringer et al., 2013). Em Mic, nesse grupo, tal parâmetro veio a se normalizar devido, provavelmente, à indução anestésica com cetamina, a qual aumenta a frequência cardíaca (Lorentz e Viana, 2011), por ação simpatomimética, voltando posteriormente a reduzir devido à ação da detomidina, havendo, inclusive, diferença significativa entre Mmpa e Mic. Já no GDD, não houve variação estatística quanto a tal parâmetro.

\begin{abstract}
Vale ressaltar que no GCD alguns animais apresentaram taquicardia, em Mbasal, devido ao estresse durante a aferição dos parâmetros basais, daí a maior variação estatística observada nesse grupo. Foi observada bradicardia em Mmpa no GCD e de Mmpa a M10 no GDD, sendo esse efeito comum quando da administração de agonistas $\alpha 2$-adrenégicos. Para o parâmetro de FC, foram considerados os valores de referência citados por Goodwin (2002) que variam de 70 a $160 \mathrm{bpm}$. Não houve diferença entre grupos em nenhum momento, mostrando que tanto a cetamina racêmica quanto a dextrocetamina influenciaram de forma similar a frequência cardíaca, concordando com Souza et al. (2002), que sugeriram que as respostas hemodinâmicas da cetamina e de seu isômero $S(+)$ são equivalentes.
\end{abstract}

Tabela 1. Média e desvio-padrão da frequência cardíaca (FC) e da pressão arterial média (PAM) e mediana e desvio interquartílico da frequência respiratória $(f)$ e da temperatura corporal (TC) de cadelas, sem raça definida, submetidas à anestesia intravenosa contínua com cetamina racêmica $(20 \mathrm{mg} / \mathrm{kg} / \mathrm{h})(\mathrm{GCD})$ ou dextrocetamina $(14 \mathrm{mg} / \mathrm{kg} / \mathrm{h})(\mathrm{GDD})$, ambas associadas à detomidina $(30 \mu \mathrm{g} / \mathrm{kg} / \mathrm{h})$

\begin{tabular}{|c|c|c|c|c|c|c|c|c|}
\hline M & \multicolumn{2}{|c|}{$\mathrm{FC}(\mathrm{bpm})$} & \multicolumn{2}{|c|}{ PAM (mmHg) } & \multicolumn{2}{|c|}{$f(\mathrm{mpm})$} & \multicolumn{2}{|c|}{$\mathrm{TC}\left({ }^{\circ} \mathrm{C}\right)$} \\
\hline & GCD & GDD & GCD & GDD & GCD & GDD & GCD & GDD \\
\hline \multirow{2}{*}{ Basal } & $140 \pm$ & $134 \pm$ & $96 \pm$ & $93 \pm$ & $31 \pm$ & $31 \pm$ & $39,1 \pm$ & $38,8 \pm$ \\
\hline & $35^{\mathrm{Aa}}$ & $26^{\mathrm{Aa}}$ & $4^{\mathrm{Aa}}$ & $9^{\mathrm{Aa}}$ & $3^{\mathrm{Aa}}$ & $3^{\mathrm{Aa}}$ & $0,2^{\mathrm{ABa}}$ & $0,8^{\mathrm{Aa}}$ \\
\hline \multirow{2}{*}{ Mpa } & $71 \pm$ & $92 \pm$ & $89 \pm$ & $99 \pm$ & $24 \pm$ & $34 \pm$ & $39,0 \pm$ & $38,5 \pm$ \\
\hline & $27^{\mathrm{Ba}}$ & $44^{\mathrm{Aa}}$ & $19^{\mathrm{Aa}}$ & $19^{\mathrm{ABa}}$ & $13^{\mathrm{Aa}}$ & $23^{\mathrm{ABa}}$ & $0,4^{\mathrm{Aa}}$ & $0,5^{\mathrm{ABb}}$ \\
\hline \multirow{2}{*}{ Mic } & $114 \pm$ & $110 \pm$ & $124 \pm$ & $130 \pm$ & $16 \pm$ & $14 \pm$ & $38,8 \pm$ & $38,5 \pm$ \\
\hline & $25^{\mathrm{Aa}}$ & $25^{\mathrm{Aa}}$ & $11^{\mathrm{Ba}}$ & $21^{\mathrm{Ba}}$ & $12^{\mathrm{ABa}}$ & $8^{\mathrm{ABa}}$ & $0,4^{\mathrm{ABa}}$ & $0,3^{\mathrm{ABb}}$ \\
\hline \multirow{2}{*}{ M10 } & $85 \pm$ & $107 \pm$ & $118 \pm$ & $126 \pm$ & $12 \pm$ & $10 \pm$ & $38,6 \pm$ & $38,3 \pm$ \\
\hline & $20^{\mathrm{Ba}}$ & $31^{\mathrm{Aa}}$ & $14^{\mathrm{Ca}}$ & $20^{\mathrm{Ba}}$ & $3^{\mathrm{Ba}}$ & $4^{\mathrm{Ba}}$ & $0,3^{\mathrm{ABCa}}$ & $0,3^{\mathrm{ABCb}}$ \\
\hline \multirow{2}{*}{ M20 } & $94 \pm$ & $106 \pm$ & $114 \pm$ & $125 \pm$ & $12 \pm$ & $12 \pm$ & $38,5 \pm$ & $38,2 \pm$ \\
\hline & $22^{\mathrm{Ba}}$ & $29^{\mathrm{Aa}}$ & $14^{\mathrm{ABCa}}$ & $18^{\mathrm{Ba}}$ & $4^{\mathrm{Ba}}$ & $6^{\mathrm{Ba}}$ & $0,5^{\mathrm{ABCa}}$ & $0,2^{\mathrm{ABCb}}$ \\
\hline \multirow{2}{*}{ M30 } & $94 \pm$ & $101 \pm$ & $110 \pm$ & $124 \pm$ & $12 \pm$ & $12 \pm$ & $38,4 \pm$ & $38,0 \pm$ \\
\hline & $23^{\mathrm{Ba}}$ & $31^{\mathrm{Aa}}$ & $14^{\mathrm{ABCa}}$ & $18^{\mathrm{Ba}}$ & $3^{\mathrm{Ba}}$ & $3^{\mathrm{Ba}}$ & $0,4^{\mathrm{ABCa}}$ & $0,3^{\mathrm{ABCb}}$ \\
\hline \multirow{2}{*}{ M40 } & $94 \pm$ & $106 \pm$ & $113 \pm$ & $117 \pm$ & $12 \pm$ & $12 \pm$ & $38,2 \pm$ & $37,9 \pm$ \\
\hline & $23^{\mathrm{Ba}}$ & $29^{\mathrm{Aa}}$ & $15^{\mathrm{ABCa}}$ & $16^{\mathrm{ABa}}$ & $5^{\mathrm{ABa}}$ & $9^{\mathrm{ABa}}$ & $0,7^{\mathrm{ABCa}}$ & $0,4^{\mathrm{ABCa}}$ \\
\hline \multirow{2}{*}{ M50 } & $98 \pm$ & $108 \pm$ & $107 \pm$ & $117 \pm$ & $12 \pm$ & $12 \pm$ & $38,1 \pm$ & $38,0 \pm$ \\
\hline & $22^{\mathrm{ABa}}$ & $26^{\mathrm{Aa}}$ & $18^{\mathrm{ABCa}}$ & $15^{\mathrm{ABa}}$ & $5^{\mathrm{ABa}}$ & $3^{\mathrm{ABa}}$ & $0,4^{\mathrm{ABCa}}$ & $0,5^{\mathrm{ABCa}}$ \\
\hline \multirow{2}{*}{ M60 } & $96 \pm$ & $108 \pm$ & $111 \pm$ & $120 \pm$ & $11 \pm$ & $15 \pm$ & $38,0 \pm$ & $37,8 \pm$ \\
\hline & $26^{\mathrm{Ba}}$ & $25^{\mathrm{Aa}}$ & $17^{\mathrm{ABCa}}$ & $20^{\mathrm{ABa}}$ & $2^{\mathrm{Ba}}$ & $7^{\mathrm{ABa}}$ & $0,5^{\mathrm{BCa}}$ & $0,6^{\mathrm{ABCa}}$ \\
\hline \multirow{2}{*}{ M70 } & $102 \pm$ & $112 \pm$ & $112 \pm$ & $115 \pm$ & $11 \pm$ & $12 \pm$ & $37,9 \pm$ & $37,9 \pm$ \\
\hline & $20^{\mathrm{ABa}}$ & $24^{\mathrm{Aa}}$ & $16^{\mathrm{ABCa}}$ & $24^{\mathrm{ABa}}$ & $2^{\mathrm{Ba}}$ & $3^{\mathrm{ABa}}$ & $0,4^{\mathrm{Ca}}$ & $0,8^{\mathrm{ABCa}}$ \\
\hline \multirow{2}{*}{ M80 } & $111 \pm$ & $112 \pm$ & $111 \pm$ & $118 \pm$ & $14 \pm$ & $15 \pm$ & $38,0 \pm$ & $37,5 \pm$ \\
\hline & $22^{\mathrm{Aa}}$ & $22^{\mathrm{Aa}}$ & $16^{\mathrm{ABCa}}$ & $20^{\mathrm{ABa}}$ & $4^{\mathrm{ABa}}$ & $5^{\mathrm{ABa}}$ & $0,4^{\mathrm{Ca}}$ & $0,9^{\mathrm{BCa}}$ \\
\hline \multirow{2}{*}{ M90 } & $108 \pm$ & $112 \pm$ & $104 \pm$ & $116 \pm$ & $12 \pm$ & $12 \pm$ & $37,8 \pm$ & $37,5 \pm$ \\
\hline & $21^{\mathrm{Aa}}$ & $24^{\mathrm{Aa}}$ & $19^{\mathrm{ACa}}$ & $14^{\mathrm{ABa}}$ & $4^{\mathrm{ABa}}$ & $4^{\mathrm{ABa}}$ & $0,7^{\mathrm{Ca}}$ & $0,8^{\mathrm{Ca}}$ \\
\hline \multirow{2}{*}{ M120 } & $102 \pm$ & $110 \pm$ & $109 \pm$ & $115 \pm$ & $22 \pm$ & $20 \pm$ & $37,8 \pm$ & $37,2 \pm$ \\
\hline & $21^{\mathrm{ABa}}$ & $18^{\mathrm{Aa}}$ & $18^{\mathrm{ABa}}$ & $17^{\mathrm{ABa}}$ & $13^{\mathrm{ABa}}$ & $10^{\mathrm{Aa}}$ & $0,6^{\mathrm{Ca}}$ & $1,3^{\mathrm{ABCa}}$ \\
\hline
\end{tabular}

$\mathrm{M}$ - momentos; bpm - batimento por minuto; $\mathrm{mmHg}$ - milímetros de mercúrio; mpm - movimento por minuto; ${ }^{\circ} \mathrm{C}$ graus Celsius. ${ }^{\mathrm{A}}$ - Em cada coluna, letras maiúsculas iguais indicam ausência de diferença estatística entre momentos dentro de cada grupo. ${ }^{\text {a }}$ - Em cada linha, letras minúsculas iguais indicam ausência de diferença estatística entre grupos em cada momento experimental. 
A PAM aumentou em Mic e M10 em relação a Mbasal e Mmpa no GCD e de Mic a M30 em comparação ao basal no GDD, não havendo diferença estatística entre grupos (Tab. 1). Esse aumento observado em ambos os grupos a partir do início da infusão contínua se deve à ação simpatomimética da cetamina (Lorentz e Viana, 2011). A ausência de diferença significativa entre grupos demonstra a equivalência entre a cetamina racêmica e a dextrocetamina quanto às respostas hemodinâmicas (Souza et al., 2002). Não ocorreu hipo ou hipertensão, considerando-se os valores médios encontrados no estudo realizado por Brown et al. (2007).

A $f$ diminuiu significativamente de M10 a M30 em relação aos valores basais em ambos os grupos (Tab. 1), havendo ainda no GCD diferença estatística em M60 e M70 em relação a Mbasal, e entre os mesmos momentos em relação a Mmpa. Nos dois grupos, ocorreu bradipneia em alguns animais a partir do Mmpa, considerando-se os valores de referência citados por Feitosa (2014) para tal parâmetro (18 a 36mpm). A depressão respiratória observada pode ser atribuível à detomidina (Ringer et al., 2013) e à cetamina, uma vez que, quando associada a fármacos depressores do SNC, ela pode causar hipoventilação e hipercapnia (Oklu et al., 2003). Não houve diferença entre grupos em nenhum momento quanto a tal parâmetro, demonstrando que a cetamina racêmica e a dextrocetamina interferiram de modo similar sobre o sistema respiratório.

Houve uma diminuição na TC de M70 a M120 em relação ao Mbasal, Mmpa e Mic no GCD. No GDD essa variável apresentou-se estatisticamente menor em M80 e M90 em relação ao Mbasal e em M90 quando comparado a Mmpa e Mic (Tab. 1). Esse efeito tem sido relatado em diversos estudos em que se utilizou anestesia dissociativa em infusão contínua (Silva et al., 2007; Henrique et al., 2019). O agonista $\alpha 2$-adrenérgico pode ter contribuído para a redução da temperatura devido à ativação dos receptores $\alpha 2-\mathrm{C}$, os quais estão relacionados aos efeitos ansiolíticos e de queda de temperatura promovidos por esses fármacos (Valverde, 2010). Não foi utilizado qualquer tipo de aquecimento, no intuito de verificar o efeito real de cada protocolo sobre esse parâmetro.

Houve diferença significativa entre grupos de Mmpa a M30, nos quais a TC apresentou-se menor no GDD quando comparada à do GCD. Essa diferença observada entre grupos pode ter ocorrido devido à hipertermia apresentada por um animal do GCD, em Mbasal e Mmpa, e por outro durante todo o período experimental. A hipertermia observada no Mbasal pode ser atribuída ao estresse causado durante a manipulação e o transporte do animal do canil ao local de experimentação. Os valores de referência para temperatura corporal foram baseados em Feitosa (2014), que os citam como de 37,8 a $39,2^{\circ} \mathrm{C}$. Não houve diferença significativa intra ou intergrupos quanto à duração e amplitude da onda P (Tab. 2 e 3), estando os valores dentro do padrão de referência para a espécie (Goodwin, 2002), o que demonstra que ambos os protocolos não interferiram no tempo de condução ou na intensidade do impulso elétrico atrial.

A duração do complexo QRS não variou entre momentos nem entre grupos (Tab. 2), permanecendo os valores dentro da referência para cães (Goodwin, 2002), em que se verificou uma ausência de interferência sobre o tempo de despolarização ventricular. Quanto à amplitude da onda R no GCD, ocorreu diferença significativa apenas entre M80 e Mmpa. Já no GDD, esse parâmetro aumentou significativamente em Mic e de M20 a M120 em relação a Mbasal (Tab. 3), mantendo-se, porém, dentro do padrão de normalidade (Goodwin, 2002), o que reflete uma influência da dextrocetamina sobre a força contrátil ventricular, que interfere na intensidade de despolarização dos ventrículos. Não houve diferença estatística ente grupos em nenhum momento quanto a essa variável (Tab. 3), mostrando que a dextrocetamina promove efeitos similares àqueles da cetamina racêmica.

Houve um aumento no intervalo QT de Mmpa a M120 em relação ao Mbasal no GCD (Tab. 2), porém os valores médios permaneceram dento dos níveis de referência (Goodwin, 2002). O intervalo QT tende a aumentar em caso de baixa frequência cardíaca, como observado no presente estudo, no qual foi observada redução de tal parâmetro nesse grupo. Essa variável eletrocardiográfica não variou significativamente no GDD, o que se deve à ausência de diferença observada também na FC. Não ocorreu diferença entre os grupos, ressaltando-se a similaridade entre as cetaminas sobre a condução elétrica cardíaca. 
Tabela 2. Mediana e desvio interquartílico dos parâmetros eletrocardiográficos de duração da onda P e do complexo QRS e média e desvio-padrão da duração do intervalo PR e QT, em milissegundos, de cadelas, sem raça definida, submetidas à anestesia intravenosa contínua com cetamina racêmica $(20 \mathrm{mg} / \mathrm{kg} / \mathrm{h})(\mathrm{GCD})$ ou dextrocetamina (14mg/kg/h) (GDD), ambas associadas à detomidina $(30 \mu \mathrm{g} / \mathrm{kg} / \mathrm{h})$

\begin{tabular}{|c|c|c|c|c|c|c|c|c|}
\hline Momentos & \multicolumn{2}{|c|}{ Onda P } & \multicolumn{2}{|c|}{ Intervalo PR } & \multicolumn{2}{|c|}{ Complexo QRS } & \multicolumn{2}{|c|}{ Intervalo QT } \\
\hline & GCD & GDD & GCD & GDD & GCD & GDD & GCD & GDD \\
\hline Mbasal & $40 \pm 4^{\mathrm{Aa}}$ & $40 \pm 1^{\mathrm{Aa}}$ & $82 \pm 14^{\mathrm{Aa}}$ & $89 \pm 12^{\mathrm{Aa}}$ & $40 \pm 5^{\mathrm{Aa}}$ & $40 \pm 1^{\mathrm{Aa}}$ & $193 \pm 18^{\mathrm{Aa}}$ & $201 \pm 27^{\mathrm{Aa}}$ \\
\hline Mmpa & $40 \pm 0^{\mathrm{Aa}}$ & $40 \pm 4^{\mathrm{Aa}}$ & $114 \pm 26^{\mathrm{Ba}}$ & $107 \pm 25^{\mathrm{Aa}}$ & $42 \pm 4^{\mathrm{Aa}}$ & $40 \pm 5^{\mathrm{Aa}}$ & $233 \pm 8^{\mathrm{BCa}}$ & $227 \pm 22^{\mathrm{Aa}}$ \\
\hline $\mathrm{Mic}$ & $42 \pm 4^{\mathrm{Aa}}$ & $42 \pm 4^{\mathrm{Aa}}$ & $104 \pm 19^{\mathrm{ABa}}$ & $114 \pm 29^{\mathrm{Aa}}$ & $40 \pm 1^{\mathrm{Aa}}$ & $42 \pm 4^{\mathrm{Aa}}$ & $212 \pm 10^{\mathrm{Ba}}$ & $220 \pm 17^{\mathrm{Aa}}$ \\
\hline M10 & $42 \pm 4^{\mathrm{Aa}}$ & $42 \pm 4^{\mathrm{Aa}}$ & $112 \pm 18^{\mathrm{Ba}}$ & $109 \pm 19^{\mathrm{Aa}}$ & $40 \pm 5^{\mathrm{Aa}}$ & $40 \pm 2^{\mathrm{Aa}}$ & $223 \pm 11^{\mathrm{BCa}}$ & $226 \pm 20^{\mathrm{Aa}}$ \\
\hline M20 & $40 \pm 1^{\mathrm{Aa}}$ & $44 \pm 4^{\mathrm{Aa}}$ & $107 \pm 19^{\mathrm{ABa}}$ & $112 \pm 29^{\mathrm{Aa}}$ & $44 \pm 4^{\mathrm{Aa}}$ & $40 \pm 5^{\mathrm{Aa}}$ & $229 \pm 14^{\mathrm{Ca}}$ & $227 \pm 17^{\mathrm{Aa}}$ \\
\hline M30 & $40 \pm 4^{\mathrm{Aa}}$ & $44 \pm 4^{\mathrm{Aa}}$ & $116 \pm 26^{\mathrm{Ba}}$ & $109 \pm 22^{\mathrm{Aa}}$ & $42 \pm 4^{\mathrm{Aa}}$ & $40 \pm 4^{\mathrm{Aa}}$ & $228 \pm 14^{\mathrm{Ca}}$ & $229 \pm 20^{\mathrm{Aa}}$ \\
\hline M40 & $40 \pm 1^{\mathrm{Aa}}$ & $44 \pm 5^{\mathrm{Aa}}$ & $109 \pm 22^{\mathrm{ABa}}$ & $116 \pm 31^{\mathrm{Aa}}$ & $42 \pm 4^{\mathrm{Aa}}$ & $40 \pm 1^{\mathrm{Aa}}$ & $227 \pm 12^{\mathrm{Ca}}$ & $227 \pm 17^{\mathrm{Aa}}$ \\
\hline M50 & $40 \pm 0^{\mathrm{Aa}}$ & $42 \pm 4^{\mathrm{Aa}}$ & $107 \pm 21^{\mathrm{ABa}}$ & $107 \pm 25^{\mathrm{Aa}}$ & $42 \pm 4^{\mathrm{Aa}}$ & $44 \pm 1^{\mathrm{Aa}}$ & $228 \pm 9^{\mathrm{Ca}}$ & $228 \pm 16^{\mathrm{Aa}}$ \\
\hline M60 & $42 \pm 4^{\mathrm{Aa}}$ & $40 \pm 4^{\mathrm{Aa}}$ & $105 \pm 16^{\mathrm{ABa}}$ & $109 \pm 24^{\mathrm{Aa}}$ & $44 \pm 4^{\mathrm{Aa}}$ & $42 \pm 4^{\mathrm{Aa}}$ & $228 \pm 14^{\mathrm{Ca}}$ & $227 \pm 18^{\mathrm{Aa}}$ \\
\hline M70 & $40 \pm 1^{\mathrm{Aa}}$ & $40 \pm 1^{\mathrm{Aa}}$ & $101 \pm 16^{\mathrm{ABa}}$ & $104 \pm 20^{\mathrm{Aa}}$ & $44 \pm 0^{\mathrm{Aa}}$ & $42 \pm 4^{\mathrm{Aa}}$ & $227 \pm 12^{\mathrm{Ca}}$ & $225 \pm 14^{\mathrm{Aa}}$ \\
\hline M80 & $40 \pm 4^{\mathrm{Aa}}$ & $44 \pm 4^{\mathrm{Aa}}$ & $100 \pm 20^{\mathrm{ABa}}$ & $103 \pm 21^{\mathrm{Aa}}$ & $44 \pm 4^{\mathrm{Aa}}$ & $44 \pm 1^{\mathrm{Aa}}$ & $226 \pm 10^{\mathrm{Ca}}$ & $227 \pm 16^{\mathrm{Aa}}$ \\
\hline M90 & $40 \pm 4^{\mathrm{Aa}}$ & $40 \pm 1^{\mathrm{Aa}}$ & $101 \pm 21^{\mathrm{ABa}}$ & $98 \pm 16^{\mathrm{Aa}}$ & $44 \pm 1^{\mathrm{Aa}}$ & $44 \pm 4^{\mathrm{Aa}}$ & $227 \pm 15^{\mathrm{Ca}}$ & $225 \pm 16^{\mathrm{Aa}}$ \\
\hline M120 & $44 \pm 5^{\mathrm{Aa}}$ & $42 \pm 4^{\mathrm{Aa}}$ & $104 \pm 17^{\mathrm{ABa}}$ & $102 \pm 13^{\mathrm{Aa}}$ & $44 \pm 4^{\mathrm{Aa}}$ & $44 \pm 4^{\mathrm{Aa}}$ & $229 \pm 16^{\mathrm{Ca}}$ & $220 \pm 23^{\mathrm{Aa}}$ \\
\hline
\end{tabular}

A - Em cada coluna, letras maiúsculas iguais indicam ausência de diferença estatística entre momentos dentro de cada grupo. ${ }^{\text {a }}$ Em cada linha, letras minúsculas iguais indicam ausência de diferença estatística entre grupos em cada momento experimental.

Tabela 3. Mediana e desvio interquartílico da amplitude da onda P e média e desvio-padrão da amplitude da onda $\mathrm{R}$, em milivolts, de cadelas, sem raça definida, submetidas à anestesia intravenosa contínua com cetamina racêmica $(20 \mathrm{mg} / \mathrm{kg} / \mathrm{h})(\mathrm{GCD})$ ou dextrocetamina $(14 \mathrm{mg} / \mathrm{kg} / \mathrm{h})(\mathrm{GDD})$, ambas associadas à detomidina $(30 \mu \mathrm{g} / \mathrm{kg} / \mathrm{h})$

\begin{tabular}{lcccc}
\multicolumn{1}{c}{ Momentos } & \multicolumn{2}{c}{ Amplitude da onda P } & \multicolumn{2}{c}{ Amplitude da onda R } \\
Mbasal & GCD & GDD & GCD & GDD \\
Mmpa & $0,24 \pm 0,05^{\mathrm{Aa}}$ & $0,22 \pm 0,03^{\mathrm{Aa}}$ & $1,25 \pm 0,53^{\mathrm{ABa}}$ & $1,18 \pm 0,34^{\mathrm{Aa}}$ \\
Mic & $0,20 \pm 0,03^{\mathrm{Aa}}$ & $0,20 \pm 0,03^{\mathrm{Aa}}$ & $1,33 \pm 0,59^{\mathrm{Aa}}$ & $1,21 \pm 0,51^{\mathrm{ABa}}$ \\
M10 & $0,18 \pm 0,02^{\mathrm{Aa}}$ & $0,19 \pm 0,04^{\mathrm{Aa}}$ & $1,42 \pm 0,50^{\mathrm{ABa}}$ & $1,35 \pm 0,49^{\mathrm{BCa}}$ \\
M20 & $0,20 \pm 0,01^{\mathrm{Aa}}$ & $0,22 \pm 0,06^{\mathrm{Aa}}$ & $1,51 \pm 0,56^{\mathrm{ABa}}$ & $1,30 \pm 0,45^{\mathrm{ABCa}}$ \\
M30 & $0,20 \pm 0,02^{\mathrm{Aa}}$ & $0,22 \pm 0,03^{\mathrm{Aa}}$ & $1,51 \pm 0,60^{\mathrm{ABa}}$ & $1,37 \pm 0,43^{\mathrm{Ca}}$ \\
M40 & $0,20 \pm 0,03^{\mathrm{Aa}}$ & $0,22 \pm 0,05^{\mathrm{Aa}}$ & $1,52 \pm 0,56^{\mathrm{ABa}}$ & $1,32 \pm 0,47^{\mathrm{Ba}}$ \\
M50 & $0,22 \pm 0,02^{\mathrm{Aa}}$ & $0,22 \pm 0,08^{\mathrm{Aa}}$ & $1,53 \pm 0,56^{\mathrm{ABa}}$ & $1,37 \pm 0,44^{\mathrm{Ca}}$ \\
M60 & $0,20 \pm 0,04^{\mathrm{Aa}}$ & $0,22 \pm 0,08^{\mathrm{Aa}}$ & $1,56 \pm 0,55^{\mathrm{ABa}}$ & $1,35 \pm 0,46^{\mathrm{Ca}}$ \\
M70 & $0,20 \pm 0,05^{\mathrm{Aa}}$ & $0,22 \pm 0,07^{\mathrm{Aa}}$ & $1,54 \pm 0,58^{\mathrm{ABa}}$ & $1,43 \pm 0,46^{\mathrm{Ca}}$ \\
M80 & $0,22 \pm 0,02^{\mathrm{Aa}}$ & $0,23 \pm 0,04^{\mathrm{Aa}}$ & $1,54 \pm 0,57^{\mathrm{ABa}}$ & $1,41 \pm 0,44^{\mathrm{Ca}}$ \\
M90 & $0,21 \pm 0,02^{\mathrm{Aa}}$ & $0,22 \pm 0,04^{\mathrm{Aa}}$ & $1,58 \pm 0,58^{\mathrm{Ba}}$ & $1,44 \pm 0,47^{\mathrm{Ca}}$ \\
M120 & $0,19 \pm 0,04^{\mathrm{Aa}}$ & $0,22 \pm 0,06^{\mathrm{Aa}}$ & $1,54 \pm 0,56^{\mathrm{ABa}}$ & $1,39 \pm 0,46^{\mathrm{Ca}}$ \\
\hline A & $0,19 \pm 0,08^{\mathrm{Aa}}$ & $0,22 \pm 0,02^{\mathrm{Aa}}$ & $1,46 \pm 0,51^{\mathrm{ABa}}$ & $1,35 \pm 0,41^{\mathrm{Ca}}$
\end{tabular}

A - Em cada coluna, letras maiúsculas iguais indicam ausência de diferença estatística entre momentos dentro de cada grupo. ${ }^{\text {a }}$ Em cada linha, letras minúsculas iguais indicam ausência de diferença estatística entre grupos em cada momento experimental.

No GCD o intervalo PR aumentou em Mmpa, M10 e M30 quando comparados ao Mbasal (Tab. 2), porém os valores médios permaneceram dentro do padrão de referência (Goodwin, 2002). Essa variável eletrocardiográfica se comporta de maneira inversamente proporcional à FC (Goodwin, 2002). Assim, tal resultado pode ser consequência de mecanismo compensatório à redução da FC apresentada nesse grupo. Não houve diferença estatística no GDD em relação a esse parâmetro devido, provavelmente, à manutenção da frequência cardíaca com a utilização da anestesia intravenosa em estudo. Não houve diferença significativa entre grupos, mostrando que ambos os protocolos intensificaram de forma similar o retardo da condução elétrica no nodo atrioventricular. 
No grupo GCD, três animais apresentaram onda T gigante, caracterizada por uma amplitude maior que um quarto da altura da onda $\mathrm{R}$, tendo dois a manifestado de Mmpa até M90. Um dos três animais demonstrou essa alteração de Mmpa até o final do período experimental, em ambos os grupos. Vale salientar que esse mesmo animal apresentou baixa frequência cardíaca durante esses mesmos momentos, o que pode ter influenciado em tal achado, uma vez que outras alterações relacionadas a ondas T evidentes, como distúrbios de condução interventricular e cardiopatias (Goodwin, 2002), não foram detectadas nesses animais.

Foi observado bloqueio atrioventricular (BAV) de primeiro grau em três animais do grupo GCD, no qual um apresentou essa arritmia em M30 e M50, um de Mic a M120 e um em M90. Em dois animais do grupo GDD, foi observada essa arritmia, tendo um destes apresentado em M40 e um de Mmpa a M80. BAV de segundo grau Mobitz tipo II foi observado em um animal do GCD em M20 e BAV de segundo grau Mobitz tipo I em um animal em Mmpa e M20. A ocorrência de BAVs quando da administração de agonistas $\alpha 2$-adrenérgicos decorre da inibição do tônus simpático ocasionada pela redução da liberação pré-sináptica de noradrenalina (Ringer et al., 2013).

Observou-se parada sinusal em seis animais do grupo GCD e em cinco do grupo GDD. Tal alteração pode ter ocorrido devido à administração da detomidina, que causa aumento do tônus vagal, induzindo a uma incapacidade momentânea do nódulo sinoatrial em gerar o impulso elétrico. Em relação à hemogasometria (Tab. 4), no GCD, o pH diminuiu significativamente 30 (M30) e 60 (M60) minutos após o início da infusão em relação a Mbasal e Mmpa, havendo valores médios menores que os relatados pela literatura para a espécie, que variam de 7,30 a 7,42 (González e Silva, 2017). No grupo GDD, não houve diferença significativa entre momentos quanto ao $\mathrm{pH}$, apesar de também haver valores médios menores que os relatados para a espécie a partir do M30. Tal diminuição nesses momentos pode estar relacionada à depressão respiratória causada pelos fármacos administrados.

Tabela 4. Média e desvio-padrão de $\mathrm{PaO}_{2}$ (em mmHg), $\mathrm{HCO}_{3}{ }^{-}(\mathrm{em} \mathrm{mmol} / \mathrm{L}), \mathrm{TCO}_{2}$ (em mmol/L), $\mathrm{SaO}$ (em $\%)$, $\mathrm{EB}$ (em mmol/L) e pH e mediana e desvio interquartílico da $\mathrm{PaCO}_{2}$ (em mmHg) de cadelas , sem raça definida, submetidas à anestesia intravenosa contínua com cetamina racêmica $(20 \mathrm{mg} / \mathrm{kg} / \mathrm{h})(\mathrm{GCD})$ ou dextrocetamina (14mg/kg/h) (GDD), ambas associadas à detomidina $(30 \mu \mathrm{g} / \mathrm{kg} / \mathrm{h})$

\begin{tabular}{|c|c|c|c|c|c|c|c|}
\hline \multirow[t]{2}{*}{ Parâmetro } & \multirow[t]{2}{*}{ Grupo } & \multicolumn{6}{|c|}{ Momentos } \\
\hline & & Mbasal & Mmpa & M30 & M60 & M90 & M120 \\
\hline \multirow{2}{*}{$\mathrm{PaO}_{2}$} & GCD & $98 \pm 3^{\mathrm{Aa}}$ & $96 \pm 5^{\text {Aa }}$ & $91 \pm 10^{\mathrm{Aa}}$ & $92 \pm 7^{\mathrm{Aa}}$ & $92 \pm 5^{\mathrm{Aa}}$ & $93 \pm 6^{\mathrm{Aa}}$ \\
\hline & GDD & $112 \pm 32^{\mathrm{Aa}}$ & $95 \pm 8^{\mathrm{Aa}}$ & $90 \pm 7^{\mathrm{Aa}}$ & $91 \pm 9^{\mathrm{Aa}}$ & $87 \pm 10^{\mathrm{Aa}}$ & $91 \pm 15^{\mathrm{Aa}}$ \\
\hline \multirow{2}{*}{$\mathrm{PaCO}_{2}$} & GCD & $43 \pm 1^{\mathrm{Aa}}$ & $40 \pm 6^{\mathrm{Aa}}$ & $46 \pm 9^{\mathrm{Aa}}$ & $42 \pm 11^{\mathrm{Aa}}$ & $41 \pm 6^{\mathrm{Aa}}$ & $40 \pm 4^{\mathrm{Aa}}$ \\
\hline & GDD & $39 \pm 8^{\mathrm{Aa}}$ & $38 \pm 3^{\mathrm{Aa}}$ & $41 \pm 8^{\mathrm{Aa}}$ & $42 \pm 7^{\mathrm{Aa}}$ & $41 \pm 5^{\mathrm{Aa}}$ & $37 \pm 6^{\mathrm{Aa}}$ \\
\hline \multirow{2}{*}{$\mathrm{TCO}_{2}$} & GCD & $21 \pm 2 \mathrm{~A}^{\mathrm{Ba}}$ & $22 \pm 3^{\mathrm{Aa}}$ & $20 \pm 2^{\mathrm{ABa}}$ & $19 \pm 2^{\mathrm{ABa}}$ & $18 \pm 3^{\mathrm{Ba}}$ & $19 \pm 2^{\mathrm{ABa}}$ \\
\hline & GDD & $20 \pm 2^{\mathrm{ABa}}$ & $22 \pm 5^{\text {Aa }}$ & $19 \pm 2^{\mathrm{ABa}}$ & $19 \pm 3^{\mathrm{ABa}}$ & $20 \pm 3^{\mathrm{ABa}}$ & $18 \pm 2^{\mathrm{Ba}}$ \\
\hline \multirow{2}{*}{$\mathrm{SaO}_{2}$} & GCD & $96 \pm 1^{\mathrm{Aa}}$ & $96 \pm 0^{\mathrm{Aa}}$ & $94 \pm 2^{\mathrm{Aa}}$ & $95 \pm 2^{\mathrm{Aa}}$ & $95 \pm 1^{\mathrm{Aa}}$ & $95 \pm 1^{\mathrm{Aa}}$ \\
\hline & GDD & $96 \pm 1^{\mathrm{Aa}}$ & $96 \pm 1^{\mathrm{Aa}}$ & $94 \pm 2^{\mathrm{Aa}}$ & $95 \pm 2^{\mathrm{Aa}}$ & $95 \pm 2^{\mathrm{Aa}}$ & $95 \pm 3^{\mathrm{Aa}}$ \\
\hline \multirow{2}{*}{$\mathrm{HCO}_{3}^{-}$} & GCD & $19 \pm 2^{\mathrm{Aa}}$ & $20 \pm 2^{\text {Aa }}$ & $19 \pm 2^{\mathrm{Aa}}$ & $17 \pm 2^{\mathrm{Aa}}$ & $17 \pm 3^{\mathrm{Aa}}$ & $18 \pm 2^{\mathrm{Aa}}$ \\
\hline & GDD & $19 \pm 2^{\mathrm{ABa}}$ & $21 \pm 5^{\mathrm{Aa}}$ & $17 \pm 1^{\mathrm{ABa}}$ & $18 \pm 3^{\mathrm{ABa}}$ & $19 \pm 3^{\mathrm{ABa}}$ & $16 \pm 2^{\mathrm{Ba}}$ \\
\hline \multirow{2}{*}{ EB } & GCD & $-7,0 \pm 3,0^{\mathrm{ABa}}$ & $-3,9 \pm 2,4^{\mathrm{Aa}}$ & $-7,8 \pm 2,3^{\mathrm{ABa}}$ & $-8,9 \pm 2,1^{\mathrm{ABa}}$ & $-8,6 \pm 3,6^{\mathrm{Ba}}$ & $-8,5 \pm 4,0^{\mathrm{ABa}}$ \\
\hline & GDD & $-5,8 \pm 2,3^{\mathrm{ABa}}$ & $-4,6 \pm 6,3^{\mathrm{Aa}}$ & $-8,8 \pm 3,1^{\mathrm{ABa}}$ & $-9,0 \pm 3,3^{\mathrm{ABa}}$ & $-7,1 \pm 4,6^{\mathrm{ABa}}$ & $-9,4 \pm 1,8^{\mathrm{Ba}}$ \\
\hline \multirow{2}{*}{$\mathrm{pH}$} & GCD & $7,3 \pm 0,0^{\mathrm{Aa}}$ & $7,3 \pm 0,0^{\mathrm{Aa}}$ & $7,2 \pm 0,0^{\mathrm{Ba}}$ & $7,2 \pm 0,0^{\mathrm{Ba}}$ & $7,2 \pm 0,1^{\mathrm{ABa}}$ & $7,3 \pm 0,0^{\mathrm{ABa}}$ \\
\hline & GDD & $7,3 \pm 0,1^{\mathrm{Aa}}$ & $7,3 \pm 0,1^{\mathrm{Aa}}$ & $7,2 \pm 0,1^{\mathrm{Aa}}$ & $7,2 \pm 0,0^{\mathrm{Aa}}$ & $7,3 \pm 0,1^{\mathrm{Aa}}$ & $7,2 \pm 0,0^{\mathrm{Aa}}$ \\
\hline
\end{tabular}

A - Em cada linha, letras maiúsculas iguais indicam ausência de diferença estatística entre momentos dentro de cada grupo. ${ }^{\text {a }}$ Em cada coluna, letras minúsculas iguais indicam ausência de diferença estatística entre grupos em cada momento experimental.

Não houve diferença significativa entre momentos, em nenhum grupo, quanto à $\mathrm{PaO}_{2} \mathrm{e}$ $\mathrm{SaO}_{2}$ (Tab. 4), apesar de haver uma tendência à redução da $\mathrm{PaO}_{2}$ em ambos os grupos em relação ao valor basal. A hipoxemia é caracterizada por uma $\mathrm{SaO}_{2}$ menor que 90\% (Cortopassi e Patricio, 2014) e $\mathrm{PaO}_{2}$ menor que $80 \mathrm{mmHg}$, sendo recomendado suporte ventilatório quando esta 
atinge valores menores que $60 \mathrm{mmHg}$ (Soares, 2012). Ambas as situações não foram encontradas em nenhum momento deste experimento, demonstrando que, apesar de ter ocorrido depressão respiratória e hipoventilação em ambos os grupos, os protocolos não foram capazes de causar hipoxemia grave.

Não ocorreu diferença significativa entre momentos em nenhum grupo quanto à $\mathrm{PaCO}_{2}$, estando os valores médios dentro dos níveis de referência para cães (35 a $45 \mathrm{mmHg}$ ) (Soares, 2012), com exceção do M30 no GCD (Tab. 4). A cetamina pode causar hipercapnia quando associada a fármacos depressores do SNC (Oklu et al., 2003). $\mathrm{O}$ valor de $\mathrm{PaCO}_{2}$ discretamente alterado no M30 no GCD, juntamente com o pH reduzido em relação ao Mbasal, indica a presença de acidose respiratória leve, consequência da hipoventilação, a qual ocorreu devido à depressão respiratória.

$\mathrm{O} \mathrm{HCO}_{3}{ }^{-}$não variou significativamente entre os momentos, no GCD, porém no GDD ocorreu diminuição significativa desse parâmetro em M120, quando comparado a Mmpa, com valores médios menores do que os relatados para a espécie (18 a 24mEq/L) (González e Silva, 2017) (Tab. 4) nesse e em vários momentos, a partir do M30, em ambos os grupos. No mecanismo de excreção do dióxido de carbono, naturalmente a equação $\mathrm{CO}_{2}$ $+\mathrm{H}_{2} \mathrm{O} \leftrightarrow \mathrm{H}_{2} \mathrm{CO}_{3} \leftrightarrow \mathrm{H}^{+}+\mathrm{HCO}_{3}^{-}$desloca-se para a direita, elevando os níveis do íon hidrogênio e bicarbonato. $\mathrm{O}$ próton $\left(\mathrm{H}^{+}\right)$é retido dentro do eritrócito ligado à hemoglobina, e o bicarbonato trocado pelo cloro na membrana do eritrócito (Smith et al., 2007). Na acidose respiratória, a $\mathrm{PaCO}_{2}$ eleva-se e o pH é reduzido devido ao escape de próton $\left(\mathrm{H}^{+}\right)$para o líquido extracelular com consequente consumo do bicarbonato. Neste experimento, $\mathrm{O}_{\mathrm{HCO}_{3}}{ }^{-}$reduziu, o que indica uma ausência do mecanismo de compensação aos distúrbios respiratórios causado pelos fármacos administrados.

O EB diminuiu significativamente em M90, no GCD, e em M120, no GDD, quando comparados a Mmpa (Tab. 4). Essas diferenças podem ser decorrentes da redução do bicarbonato, a qual promoveu uma ausência de base para neutralizar ácidos naturalmente produzidos, resultando em excesso de base negativo. Os valores médios para o EB, em ambos os grupos, mantiveram-se dentro do padrão de normalidade, que varia de $-9,7$ a 2,3mmHg (Nelson e Couto, 2015), não condizendo com uma acidose metabólica.

$\mathrm{O} \mathrm{TCO}_{2}$ diminuiu em M90 quando comparado ao Mmpa no GCD e em M120 em relação ao Mmpa no GDD (Tab. 4). Considerando-se que o $\mathrm{TCO}_{2}$ mensura tanto o $\mathrm{CO}_{2}$ dissolvido no sangue quanto o $\mathrm{HCO}_{3}^{-}$presente na amostra, em indivíduos normais o $\mathrm{TCO}_{2}$ é maior do que a concentração de $\mathrm{HCO}_{3}{ }^{-}$em 1 a $2 \mathrm{mEq} / \mathrm{L}$. A redução do $\mathrm{HCO}_{3}{ }^{-}$causa diminuição no $\mathrm{TCO}_{2}$ e $\mathrm{EB}$, evento esse que foi observado no presente experimento. O uso da cetamina $\mathrm{S}(+)$ ou racêmica não interferiu nos parâmetros hemogasométricos, uma vez que não ocorreu diferença estatística entre grupos em nenhum momento (Tab. 4), porém a presença de acidose respiratória leve exige uma maior atenção ao sistema respiratório quando da utilização dos protocolos estudados.

Não houve diferença intra ou intergrupos em relação à intensidade do estímulo elétrico necessária para desencadear resposta (Tab. 5), mostrando que a cetamina $\mathrm{S}(+)$ utilizada em uma dose $30 \%$ menor que a cetamina racêmica apresenta o mesmo potencial analgésico que esta, concordando com Casoni et al. (2015), que afirmam que a cetamina $\mathrm{S}(+)$ é 1,5 vez mais potente que o racemato em cães. A intensidade da corrente aplicada na cauda variou de 10 a $35 \mathrm{~mA}$, do início da infusão até 30 minutos após o fim desta. Figueiró et al. (2016) encontraram correntes de 30 a 50mA aplicadas no mesmo sítio anatômico para determinação da concentração alveolar mínima (CAM) do isoflurano em cães.

A estimulação elétrica representa um estímulo que é frequentemente usado para determinação da CAM de anestésicos inalatórios (Seddighi et al., 2011; Figueiró et al., 2016). Esse tipo de estímulo apresenta algumas vantagens sobre o pinçamento da cauda, à medida que sua quantificação, padronização e reprodutibilidade são mais facilmente atingidas (LeBars et al., 2001). Apesar disso, ainda é questionável se um estímulo supramáximo é necessário para garantir repetibilidade de estimulação (Eger et al., 2008) e se pode haver habituação, por parte do animal, ao estímulo (Laster et al., 1993). 
Tabela 5. Média e desvio-padrão da intensidade do estímulo elétrico (em miliampéres) necessária para desencadear resposta e mediana (valor máximo - valor mínimo) dos escores de miorrelaxamento de cadelas, sem raça definida, submetidas à anestesia intravenosa contínua com cetamina racêmica $(20 \mathrm{mg} / \mathrm{kg} / \mathrm{h})(\mathrm{GCD})$ ou dextrocetamina $(14 \mathrm{mg} / \mathrm{kg} / \mathrm{h})(\mathrm{GDD})$, ambas associadas à detomidina $(30 \mu \mathrm{g} / \mathrm{kg} / \mathrm{h})$

\begin{tabular}{lcccc}
\multicolumn{1}{c}{ Momentos } & \multicolumn{2}{c}{ Estímulo Eelétrico } & \multicolumn{2}{c}{ Miorrelaxamento } \\
Mbasal & GCD & GDD & GCD & GDD \\
Mmpa & - & - & $0,0(0,0-0,0)^{\mathrm{Aa}}$ & $0,0(0,0-0,0)^{\mathrm{Aa}}$ \\
Mic & - & - & $0,0(1,0-0,0)^{\mathrm{ABa}}$ & $0,0(1,0-0,0)^{\mathrm{Aa}}$ \\
M10 & $19,4 \pm 9,4^{\mathrm{Aa}}$ & $18,1 \pm 9,2^{\mathrm{Aa}}$ & $1,0(2,0-1,0)^{\mathrm{ABa}}$ & $1,0(2,0-0,0)^{\mathrm{ABa}}$ \\
M20 & $21,9 \pm 7,5^{\mathrm{Aa}}$ & $21,3 \pm 7,9^{\mathrm{Aa}}$ & $2,0(2,0-1,0)^{\mathrm{Ba}}$ & $2,0(2,0-0,0)^{\mathrm{ABa}}$ \\
M30 & $21,9 \pm 5,9^{\mathrm{Aa}}$ & $21,9 \pm 5,9^{\mathrm{Aa}}$ & $2,0(2,0-1,0)^{\mathrm{Ba}}$ & $2,0(2,0-0,0)^{\mathrm{ABa}}$ \\
M40 & $21,9 \pm 5,9^{\mathrm{Aa}}$ & $23,1 \pm 3,7^{\mathrm{Aa}}$ & $2,0(2,0-1,0)^{\mathrm{Ba}}$ & $2,0(2,0-0,0)^{\mathrm{ABa}}$ \\
M50 & $22,5 \pm 5,3^{\mathrm{Aa}}$ & $21,9 \pm 2,6^{\mathrm{Aa}}$ & $2,0(2,0-1,0)^{\mathrm{Ba}}$ & $2,0(2,0-0,0)^{\mathrm{ABa}}$ \\
M60 & $22,5 \pm 5,3^{\mathrm{Aa}}$ & $21,9 \pm 2,6^{\mathrm{Aa}}$ & $2,0(2,0-1,0)^{\mathrm{Ba}}$ & $2,0(2,0-0,0)^{\mathrm{ABa}}$ \\
M70 & $23,1 \pm 4,6^{\mathrm{Aa}}$ & $21,9 \pm 2,6^{\mathrm{Aa}}$ & $2,0(2,0-1,0)^{\mathrm{Ba}}$ & $2,0(2,0-0,0)^{\mathrm{ABa}}$ \\
M80 & $23,1 \pm 4,6^{\mathrm{Aa}}$ & $21,9 \pm 2,6^{\mathrm{Aa}}$ & $2,0(2,0-1,0)^{\mathrm{Ba}}$ & $2,0(2,0-1,0)^{\mathrm{ABa}}$ \\
M90 & $21,9 \pm 3,7^{\mathrm{Aa}}$ & $23,1 \pm 5,3^{\mathrm{Aa}}$ & $2,0(2,0-1,0)^{\mathrm{Ba}}$ & $2,0(2,0-1,0)^{\mathrm{Ba}}$ \\
M120 & $21,3 \pm 3,5^{\mathrm{Aa}}$ & $23,1 \pm 5,3^{\mathrm{Aa}}$ & $1,5(2,0-1,0)^{\mathrm{ABa}}$ & $2,0(2,0-1,0)^{\mathrm{Ba}}$ \\
\hline
\end{tabular}

A - Em cada coluna, letras maiúsculas iguais indicam ausência de diferença estatística entre momentos dentro de cada grupo. ${ }^{\text {a }}$ Em cada linha, letras minúsculas iguais indicam ausência de diferença estatística entre grupos em cada momento experimental.

No GCD, o miorrelaxamento foi significativamente maior a partir de 10 minutos (M10) até 80 minutos (M80) (Tab. 5), em relação ao Mbasal. Nesse grupo, em Mmpa, 37,5\% dos animais apresentaram miorrelaxamento bom e 62,5\% ruim; em Mic, em 62,5\% dos animais, o miorrelaxamento foi considerado bom e em $37,5 \%$, ruim; de M10 a M40, 25\% das cadelas demonstraram relaxamento muscular bom e $75 \%$ excelente; de M50 a M80, o miorrelaxamento foi classificado em $37,5 \%$ dos animais como bom e em $62,5 \%$ como excelente; em M90, 50\% dos animais apresentaram miorrelaxamento bom e $50 \%$ excelente; e 30 minutos após o fim da infusão (M120), 37,5\% tiveram miorrelaxamento ruim, $37,5 \%$ miorrelaxamento bom e $25 \%$, excelente.

No GDD, o miorrelaxamento variou em relação aos valores basais apenas em M80 e M90, havendo ainda diferença entre esses momentos e o Mmpa (Tab. 5). Nesse grupo, em Mmpa, 37,5\% dos animais apresentaram miorrelaxamento bom e $62,5 \%$ ruim; em Mic, $12,5 \%$ manifestaram miorrelaxamento ruim, $50 \%$ bom e $37,5 \%$ excelente; de M10 a M60, 87,5\% das cadelas demonstraram miorrelaxamento excelente e em $12,5 \%$ esse foi ruim; em M70, 25\% tiveram relaxamento muscular bom e $75 \%$ excelente; em M80 e M90, $12,5 \%$ apresentaram miorrelaxamento bom e $87,5 \%$ excelente; em M120, $50 \%$ das cadelas demonstraram miorrelaxamento ruim, $25 \%$ miorrelaxamento bom e 25\%, excelente. O miorrelaxamento observado se deve ao agonista $\alpha 2$-adrenérgico administrado (Silva et al., 2007).

Não houve diferença entre grupos em relação ao período de recuperação, sendo este de 219,6 $6 \pm 72,3$ minutos no GCD e de $234,1 \pm 96,8$ minutos no GDD, demonstrando que a cetamina racêmica e a dextrocetamina interferem de forma similar nesse parâmetro. Henrique et al. (2019), ao utilizarem a anestesia intravenosa contínua com dextrocetamina e detomidina, nas mesmas doses aqui utilizadas, observaram um período de recuperação médio de 302 minutos, não havendo dados na literatura sobre o período de recuperação da administração de cetamina racêmica associada à detomidina por via intravenosa contínua. Larenza et al. (2008) afirmam que, se administrada sozinha, a cetamina $\mathrm{S}(+)$ apresenta um clearence maior do que a mistura racêmica, resultando em eliminação e recuperação mais rápidas. Acredita-se que a detomidina possa ter aumentado o tempo de recuperação.

A qualidade da recuperação no GCD foi classificada como boa em seis animais, nos quais se observou discreta agitação e vocalização, opistótono, nistagmo, rigidez muscular, vômito e mioclonias. Os demais animais deste grupo apresentaram recuperação excelente. No GDD, dois animais apresentaram recuperação excelente; 
cinco animais tiveram recuperação boa, manifestando discreta agitação, vocalização, salivação, opistótono e rigidez muscular; e uma cadela apresentou recuperação ruim, com salivação excessiva, excitação, rigidez muscular, opistótono, defecação e micção. A presença de rigidez muscular observada em ambos os grupos se deve à administração de cetamina, que aumenta o tônus muscular. Apesar de ter sido utilizada a detomidina, potente miorrelaxante, segundo Valadão (2019) a coadministração de substâncias biotransformadas no fígado pode aumentar a meia-vida da cetamina, em razão da competição enzimática, como no presente caso, em que, provavelmente, o efeito miorrelaxante da detomidina apresentou uma menor duração em comparação ao aumento do tônus muscular promovido pelas cetaminas.

Além disso, as doses utilizadas no presente estudo podem ter influenciado o maior efeito da cetamina sobre o miorrelaxamento promovido pela detomidina. Os outros efeitos indesejáveis observados são comuns quando da utilização de anestésicos dissociativos. Embora a maioria dos estudos afirmem que a intensidade e ocorrência de reações indesejáveis durante a recuperação anestésica é menor com o uso da cetamina $\mathrm{S}(+)$ (Pfenninger et al., 2002), no presente estudo houve similaridade entre as reações observadas em ambos os grupos.

\section{CONCLUSÕES}

A cetamina racêmica e a dextrocetamina, utilizadas numa proporção aproximada de 1,5:1, e administradas associadas à detomidina pela via intravenosa contínua, causam efeitos cardiorrespiratórios e anestésicos similares, recomendando-se a suplementação com oxigênio e a monitoração eletrocardiográfica constante.

\section{REFERÊNCIAS}

BROWN, S.; ATKINS, C.; BAGLEY, R. et al. Guidelines for the identification, evaluation, and management of systemic hypertension in dogs and cats. J. Vet. Intern. Med., v.21, p.542-558, 2007.

CARDOSO, F.T.S.; FEITOSA JÚNIOR, F.S.; DINIZ, B.L.M. et al. Neuroleptoanalgesia associada à anestesia epidural com lidocaína e xilazina em cutias (Dasyprocta aguti). Acta Sci. Vet., v.36, p.149-154, 2008.
CASONI, D.; SPADAVECCHIA, C.; ADAMI, C. S-ketamine versus racemic ketamine in dogs: their relative potency as induction agents. Vet. Anaesth. Analg., v.42, p.250-259, 2015.

CORTOPASSI, S.R.G.; PATRICIO, G.C.F. Fluidoterapia na anestesia. In: FANTONI, D.T.; CORTOPASSI, S.R.G. Anestesia em cães e gatos. 2.ed. São Paulo: Roca, 2014. p.131-146.

EGER, E.I.; RAINES, D.E.; SHAFER, S.L. et al. Is a new paradigm needed to explain how inhaled anesthetics produce immobility? Vet. Anesth. Analg., v.107, p.832-848, 2008.

FEITOSA, F.L.F. Exame físico geral ou de rotina. In:_. Semiologia veterinária: $A$ arte do diagnóstico. 6.ed. São Paulo: Roca, 2014. p.5168 .

FIGUEIRÓ, M.R.; SOARES, J.H.N.; ASCOLI, F.O. et al. Isoflurane MAC determination in dogs using three intensities of constant-current electrical stimulation. Vet. Anaesth. Analg., v.43, p.464-471, 2016.

GONZÁLEZ, F.H.D.; SILVA, S.C. Introdução à bioquímica veterinária. 3.ed. Porto Alegre: UFRGS, 2017. 538p.

GOODWIN, J.K. Eletrocardiografia. In: GOODWIN, J.K.; TILLEY, L.P. Manual de cardiologia para cães e gatos. 3.ed. São Paulo: Roca, 2002. p.39-65.

HENRIQUE, F.V.; PEREIRA, S.A.R.; MEDEIROS, L.K.G. et al. Anestesia intravenosa contínua com dextrocetamina e detomidina em cadelas submetidas à ovário-histerectomia e prémedicadas com midazolam e morfina. Acta Sci. Vet., v.47, p.1650, 2019.

LARENZA, M.P.; ALTHAUS, H.; CONROT, A. et al. Anaesthesia recovery quality after racemic ketamine or S-ketamine administration to male cats undergoing neutering surgery. Schweizer Archiv Tierheilk., v.150, p.599-607, 2008.

LASTER, M.J.; LIU, J.; EGER, E.I.; TAHERI, S. Electrical stimulation as a substitute for the tail clamp in the determination of minimum alveolar concentration. Vet. Anesth. Analg., v.76, p.13101312, 1993.

LE BARS, D.; GOZARIU, M.; CADDEN, S.W. Animal models of nociception. Pharmacol. Rev., v.53, p.597-652, 2001. 
LORENTZ, M.N.; VIANA, B.S.B. Disritmias cardíacas e anestesia. Rev. Bras. Anestesiol., v.61, p.798-813, 2011.

NELSON, R.W.; COUTO, C.G. Distúrbios do pâncreas endócrino. In: Medicina interna de pequenos animais. 5.ed. São Paulo: Elsevier, 2015. p.2270-2354.

OKLU E.; BULUTCU F.S; YALCIN Y. et al. Which anesthetic agent alters the hemodynamic status during pediatric catheterization? Comparison of propofol versus ketamine. $J$. Cardiothorac. Vasc. Anesth., v.17, p.686-690, 2003.

PFENNINGER, E.G.; DURIEUX, M.E.; HIMMELSEHER, S. Cognitive impairment after small-close ketamine isomers in comparison to equianalgesic racemic ketamine in human volunteers. Anesthesiology, v.96, p.357-366, 2002.

POHL, V.H.; CARREGARO, A.B.; LOPES, C. et al. Epidural anesthesia and postoperatory analgesia with alpha-2 adrenergic agonists and lidocaine for ovariohysterectomy in bitches. Can. J. Vet. Res., v.76, p.215-220, 2012.

RINGER， S.K.; SCHWARZWALD， C.C.; PORTIER, K.G. et al. Effects on cardiopulmonary function and oxygen delivery of doses of romifidine and xylazine followed by constant rate infusions in standing horses. Vet. J., v.195, p.228-234, 2013.

SEDDIGHI, R.; EGGER, C.M.; ROHRBACH, B.W. et al. The effect of midazolam on the endtidal concentration of isoflurane necessary to prevent movement in dogs. Vet. Anaesth. Analg., v.38, p.195-202, 2011.
SILVA, F.C.; HATSCHBACH, E.; LIMA, A.F.M.; CARVALHO, Y.K. Continuous infusion in adult females dogs submitted to ovariohysterectomy with midazolam-xylazine and/or medetomidine pre-treated with methotrimeprazine and buprenorphine. Acta Cir. Bras., v.22, p.272-278, 2007.

SMITH, C.; MARKS, A.D.; LIEBERMAN, M. Bioquímica médica básica de Marks: uma abordagem clínica. 2.ed. Porto Alegre: Artmed, 2007. 992p.

SOARES, J.H.N. Fisiologia respiratória e ventilatória. In: RABELO, R. Emergências de pequenos animais. Rio de Janeiro: Elsevier, 2012. p.857-867.

SOUZA, A.P.; CARARETO, R.; NUNES, N. et $a l$. Eletrocardiografia em cães anestesiados com cetamina-S ou cetamina. Ciênc. Rural, v.32, p.787-791, 2002.

VALADÃO, C.A.A. Anestesia dissociativa. In: MASSONE, F. Anestesiologia veterinária farmacologia e técnicas. 7.ed. Rio de Janeiro: Guanabara Koogan, 2019. p.53-62.

VALVERDE, A. Alpha-2 agonists as pain therapy in horses. Vet. Clinic. N. Am. Equine Pract., v.26, p.515-532, 2010. 